Geometry 85 Topology

Volume 7 (2003) 615-639

Published: 22 October 2003

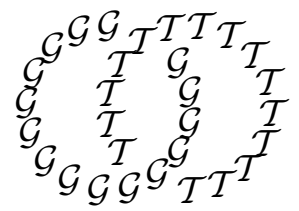

\title{
Knot Floer homology and the four-ball genus
}

\author{
Peter Ozsváth \\ ZoLtÁn SzaBó \\ Department of Mathematics, Columbia University \\ New York 10025, USA \\ and \\ Department of Mathematics, Princeton University \\ New Jersey 08540, USA
}

Email: petero@math.columbia.edu and szabo@math.princeton.edu

\begin{abstract}
We use the knot filtration on the Heegaard Floer complex $\widehat{C F}$ to define an integer invariant $\tau(K)$ for knots. Like the classical signature, this invariant gives a homomorphism from the knot concordance group to $\mathbb{Z}$. As such, it gives lower bounds for the slice genus (and hence also the unknotting number) of a knot; but unlike the signature, $\tau$ gives sharp bounds on the four-ball genera of torus knots. As another illustration, we calculate the invariant for several ten-crossing knots.
\end{abstract}

AMS Classification numbers Primary: 57R58

Secondary: 57M25, 57M27

Keywords: Floer homology, knot concordance, signature, 4-ball genus

Proposed: Robion Kirby

Seconded: Tomasz Mrowka, Cameron Gordon
Received: 16 January 2003

Revised: 17 October 2003

(c) Geometry $8 \mathcal{G}$ Topology $\mathcal{P}$ ublications 


\section{Introduction}

Let $K$ be a knot in the three-sphere. A slice surface for $K$ is a smooth submanifold-with-boundary in $B^{4}$ whose boundary is the knot $K$. The fourball genus $g^{*}(K)$ is the minimal genus of any slice surface for $K$. The four-ball genus gives a lower bound for the "unknotting number" $u(K)$ (the minimal number of crossing-changes required to unknot $K$ ).

Our aim here is to define an integer invariant of a knot $K$ which gives a lower bound on $g^{*}(K)$, using the knot filtration from [17], see also [22] and [21]. Specifically, letting $\widehat{C F}\left(S^{3}\right)$ denote the chain complex whose homology calculates the Heegaard Floer homology $\widehat{H F}\left(S^{3}\right)$ (cf. [15], see also Section 2 for a quick explanation), recall that a knot $K$ in $S^{3}$ induces a filtration on the chain complex $\widehat{C F}\left(S^{3}\right)$. Letting $\mathcal{F}(K, m) \subset \widehat{C F}\left(S^{3}\right)$ be the subcomplex generated by intersection points whose filtration level is less than or equal to $m$, we obtain an induced sequence of maps

$$
\iota_{K}^{m}: H_{*}(\mathcal{F}(K, m)) \longrightarrow H_{*}\left(\widehat{C F}\left(S^{3}\right)\right)=\widehat{H F}\left(S^{3}\right) \cong \mathbb{Z},
$$

which are isomorphisms for all sufficiently large integers $m$. Let

$$
\tau(K)=\min \left\{m \in \mathbb{Z} \mid \iota_{K}^{m} \text { is non-trivial }\right\} .
$$

As we shall see, the invariant $\tau(K)$ in fact gives a lower bound on the following generalization of the four-ball genus. Let $W$ be a smooth, oriented fourmanifold with $\partial W=S^{3}$ and with $b_{2}^{+}(W)=b_{1}(W)=0$ (as usual $b_{1}$ denotes the first Betti number, and $b_{2}^{+}$denotes the maximum dimension of any vector subspace $V$ of $H^{2}(W)$ on which the cup-product form is positive-definite). According to Donaldson's celebrated theorem [3], the intersection form of $W$ is diagonalizable (though in the applications it is interesting to consider the special case where $W=B^{4} \#^{b} \overline{\mathbb{C P}}^{2}$, so the intersection form is already diagonalized). Writing a homology class $[\Sigma] \in H_{2}(W)$ as

$$
[\Sigma]=s_{1} \cdot e_{1}+\ldots+s_{b} \cdot e_{b},
$$

where $e_{i}$ are an ortho-normal basis for $H^{2}(W ; \mathbb{Z})$, and $s_{i} \in \mathbb{Z}$, we can define the $L^{1}$ norm of $[\Sigma]$ by

$$
|[\Sigma]|=\left|s_{1}\right|+\ldots+\left|s_{b}\right| .
$$

Note that this is independent of the diagonalization (since the basis $\left\{e_{i}\right\}$ is uniquely characterized, up to permutations and multiplications by \pm 1 , by the ortho-normality condition). We then have the following bounds on the genus of $\Sigma$, which are proved in Section 3: 
Theorem 1.1 Let $W$ be a smooth, oriented four-manifold with $b_{2}^{+}(W)=0=$ $b_{1}(W)$, and $\partial W=S^{3}$. If $\Sigma$ is any smoothly embedded surface-with-boundary in $W$ whose boundary lies on $S^{3}$, where it is embedded as the knot $K$, then we have the following inequality:

$$
2 \tau(K)+|[\Sigma]|+[\Sigma] \cdot[\Sigma] \leq 2 g(\Sigma) .
$$

The quantity $\tau(K)$ is additive under connected sums. This additivity (together with the above theorem), can be reformulated in the following terms.

Recall that two knots $K_{1}$ and $K_{2}$ are said to be concordant if there is a smoothly embedded cylinder in $[1,2] \times S^{3}$ which meets $\{i\} \times S^{3}$ in $K_{i}($ for $i=1,2)$. The connected sum of knots descends to the set of concordance classes of knots Conc $\left(S^{3}\right)$ to endow the latter object with the structure of an Abelian group. The following result is also established in Section 3:

Theorem 1.2 The map $\tau$ induces a group homomorphism from $\operatorname{Conc}\left(S^{3}\right)$ to $\mathbb{Z}$.

Indeed, by letting $W$ be the four-ball in Theorem 1.1, and reflecting $K$ if necessary, we obtain the following:

Corollary 1.3 Let $K \subset S^{3}$ be a knot. Then,

$$
|\tau(K)| \leq g^{*}(K) .
$$

There is a classical knot invariant which has many of the same properties which $\tau$ has - the signature of $K, \sigma(K)$. In fact, $\tau$ and $-\sigma / 2$ agree for a very wide class of knots. For instance, results from [19] (see also [21]) give the following:

Theorem 1.4 Let $K$ be an alternating knot in $S^{3}$. Then, $\tau(K)=-\sigma(K) / 2$.

Proof From Theorem 1.3 of [19], for an alternating knot, $H_{*}\left(\frac{\mathcal{F}(K, m))}{\mathcal{F}(K, m-1)}\right)$ is supported in dimension $m+\frac{\sigma}{2}$. The result follows immediately.

In fact, $\tau(K)=-\sigma(K) / 2$ holds for some non-alternating knots as well (compare Section 6 of $[20])$.

The following consequence of Theorems 1.1 and 1.2 proved in Section 3 underscores the similarity between $-\sigma / 2$ and $\tau$ : 
Corollary 1.5 Let $K_{+}$be a knot in $S^{3}$, and $K_{-}$be the new knot by changing one positive crossing in $K_{+}$to a negative crossing. Then,

$$
\tau\left(K_{+}\right)-1 \leq \tau\left(K_{-}\right) \leq \tau\left(K_{+}\right) .
$$

Observe that $-\sigma(K) / 2$ also satisfies the above inequality. Indeed according to $[4],-\frac{\sigma}{2}$ is characterized among integer-valued knot invariants by the following three properties:

- it vanishes for the unknot

- it satisfies inequalities corresponding to the ones for $\tau$ stated in the above corollary

- its parity is determined by the sign of $\Delta_{K}(-1)$.

However in general, $-\sigma / 2 \neq \tau$. As an example, the knot $9_{42}$ has $\tau=0$ and $\sigma=2$. (The calculation of $\tau$ is a straightforward consequence of the calculations from Proposition 6.5 of [17].) Other small examples are given in Section 4. An infinite family of examples is provided by the following result proved in [12]:

Theorem 1.6 [Corollary 1.9 of [12]] Let $K$ be a knot in $S^{3}$ and suppose that there is some integer $p \geq 0$ with the property that $S_{p}^{3}(K)=L(p, q)$ for some $q$. Then, $\tau(K)$ is the degree of the symmetrized Alexander polynomial of $K$.

Corollary 1.7 Let $p$ and $q$ a pair of positive, relatively prime integers, and let $T_{p, q}$ denote the $(p, q)$ torus knot. Then,

$$
\left|\tau\left(T_{p, q}\right)\right|=\frac{p q-p-q+1}{2} .
$$

Proof For a suitable chirality on $T_{p, q}$, we have that $p q \pm 1$-surgery on $T_{p, q}$ is a lens space. Now, apply Theorem 1.6.

Of course, the above corollary gives an infinite set of knots for which $\sigma(K) \neq$ $2 \tau(K)$ (see [5] for the calculation of the signature of torus knots). For instance, $\sigma\left(T_{5,4}\right) / 2=4$, while $\tau\left(T_{5,4}\right)=5$.

It follows quickly from Corollary 1.7 and Corollary 1.3 that the four-ball genus and indeed the unknotting number of $T_{p, q}$ is $(p q-p-q+1) / 2$, a result first proved by Kronheimer and Mrowka [8] using Donaldson's invariants [3], and conjectured by Milnor (cf. [9], see also [2], [24]). Indeed, constructions of Berge [1] give other fibered knots for which Theorem 1.6 applies. Thus, for the knots arising from Berge's constructions, the four-ball genus agrees with 
the degree of the Alexander polynomial (which, since those knots are fibered, agrees with their Seifert genus). For more on this, see [12].

We give also calculations for some ten-crossing knots in Section 4. The calculations rest on the combinatorial techniques developed in [19] and extended in [20]. These calculations can be used to determine the four-ball genera of some 10-crossing knots, whose four-ball genera were previously calculated using techniques from gauge theory (cf. [8], [24], [25], [7]).

In closing, we observe that many of the constructions from this paper can be generalized to the case of null-homologous, oriented links in a compact, oriented three-manifold. We sketch some of these generalizations in Section 5.

In the proof of Theorem 1.1, we make use of the knot filtration and its relationship with the Heegaard Floer homology of a corresponding surgered threemanifold. This relationship is spelled out in detail in Section 4 of [17]. For the reader's convenience, we review some aspects of this in Section 2 before turning to the proofs of the main result in Section 3. The ten-crossing calculations are described in Section 4.

Remarks The invariant $\tau(K)$ and also some additional constructions have been independently discovered by Rasmussen in his thesis [22]. A construction for obtaining information on the slice genus, using Heegaard Floer homology in a different way, has been developed by Strle and Owens, cf. [10]. We are also indebted to Jacob Rasmussen and Chuck Livingston for many valuable comments on an early version of this preprint.

\section{Properties of the knot filtration}

We recall here the knot filtration from [17], focusing on the case of knots in $S^{3}$. In Subsection 2.1, we recall the definition, and in Subsection 2.2 we recall its relationship with Heegaard Floer homology of the surgered three-manifold.

\subsection{Definition of the knot filtrations}

We briefly recall here the construction of the knot filtration. For simplicity, we restrict to knots $K$ in the three-sphere.

Fix a doubly-pointed Heegaard diagram $(S, \boldsymbol{\alpha}, \boldsymbol{\beta}, w, z)$ for the knot $K \subset S^{3}$, in the following sense. Here, $S$ is an oriented surface of genus $g, \boldsymbol{\alpha}=\left\{\alpha_{1}, \ldots, \alpha_{g}\right\}$ 
is a $g$ tuple of homologically linearly independent, pairwise disjoint, simple closed curves in $S$, as is $\boldsymbol{\beta}=\left\{\beta_{1}, \ldots \beta_{g}\right\}$. Of course, $\boldsymbol{\alpha}$ and $\boldsymbol{\beta}$ specify a pair of handlebodies $U_{\alpha}$ and $U_{\beta}$ which bound $S$. We require that $(S, \boldsymbol{\alpha}, \boldsymbol{\beta})$ is a Heegaard diagram for $S^{3}$, and also that the knot $K$ is supported inside $U_{\beta}$ as an unknotted circle which meets the disk attached to $\beta_{1}$ transversally in one point, and none of the other attaching attaching disks. In particular, $\beta_{1}$ represents a meridian for $K$. The two points $w$ and $z$ lie on $S-\alpha_{1}-\ldots-\alpha_{g}-\beta_{1}-\ldots-\beta_{g}$, and can be connected by an arc which meets $\beta_{1}$ exactly once, and none of the other attaching circles.

We consider the $g$-fold symmetric product $\operatorname{Sym}^{g}(S)$, with two distinguished tori

$$
\mathbb{T}_{\alpha}=\alpha_{1} \times \cdots \times \alpha_{g} \quad \text { and } \quad \mathbb{T}_{\beta}=\beta_{1} \times \cdots \times \beta_{g} .
$$

The generators $X$ for the chain complex $\widehat{C F}\left(S^{3}\right)$ are intersection points between $\mathbb{T}_{\alpha}$ and $\mathbb{T}_{\beta}$ in $\operatorname{Sym}^{g}(S)$.

Fix intersection points $\mathbf{x}, \mathbf{y} \in X$. A Whitney disk $u$ connecting $\mathbf{x}$ to $\mathbf{y}$ is a map

$$
u:\{\mathbf{z} \in \mathbb{C}|| z \mid \leq 1\} \longrightarrow \operatorname{Sym}^{g}(\Sigma)
$$

satisfying the boundary conditions

$$
\begin{aligned}
u\{\zeta \mid \operatorname{Re}(\zeta) \geq 0 \text { and }|\zeta|=1\} \subset \mathbb{T}_{\alpha}, & u\{\zeta \mid \operatorname{Re}(\zeta) \leq 0 \text { and }|\zeta|=1\} \subset \mathbb{T}_{\beta}, \\
u(-\sqrt{-1})=\mathbf{x}, & u(\sqrt{-1})=\mathbf{y} .
\end{aligned}
$$

For a fixed point $p \in S-\alpha_{1}-\cdots-\alpha_{g}-\beta_{1}-\cdots-\beta_{g}$, let $n_{p}(u)$ denote the algebraic intersection number of $u$ with the submanifold $\{p\} \times \operatorname{Sym}^{g-1}(S)$. Note that $n_{p}(u)$ depends only on the homotopy class $\phi$ of $u$. (In this context, homotopies are to be understood as homotopies of maps all of which are Whitney disks.)

There is a function

$$
\mathcal{F}: X \longrightarrow \mathbb{Z}
$$

uniquely characterized by the following two properties. For any $\mathbf{x}, \mathbf{y} \in X$, we have that

$$
\mathcal{F}(\mathbf{x})-\mathcal{F}(\mathbf{y})=n_{z}(\phi)-n_{w}(\phi),
$$

and also

$$
P(T)=\sum_{\mathbf{x} \in X} \epsilon(\mathbf{x}) \cdot T^{\mathcal{F}(\mathbf{x})}
$$

is a symmetric Laurent polynomial in the formal variable $T$, where here, $\epsilon(\mathbf{x}) \in$ $\{ \pm 1\}$ denotes the local intersection number of $\mathbb{T}_{\alpha}$ and $\mathbb{T}_{\beta}$ at $\mathbf{x}$, with respect to 
fixed orientations on the tori and $\operatorname{Sym}^{g}(S)$. (Indeed, for the appropriate choice of overall sign, $P(T)$ coincides with the symmetrized Alexander polynomial of $K$, cf. Equation (1) of [17].)

Recall [15] that there is a homology theory for (closed, oriented) three-manifolds $\widehat{H F}(Y)$, whose generators are intersection points $X$, and whose differentials count pseudo-holomorphic Whitney disks in $\operatorname{Sym}^{g}(S)$, in the homotopy class with $n_{w}(\phi)=0$. In the case where $Y \cong S^{3}, \widehat{H F}(Y) \cong \mathbb{Z}$. Starting with a doubly-pointed Heegaard diagram for $S^{3}$ compatible with a knot $K \subset S^{3}$, it is not difficult to see that if we let $\mathcal{F}(K, m) \subset \widehat{C F}\left(S^{3}\right)$ denote the subset generated by those $\mathbf{x} \in X$ with $\mathcal{F}(\mathbf{x}) \leq m$, then that subset indeed is preserved by the differential, i.e. $\mathcal{F}(K, m)$ defines a $\mathbb{Z}$-filtration of $\widehat{C F}\left(S^{3}\right)$, indexed by integers $m \in \mathbb{Z}$. We let $\widehat{C F K}\left(S^{3}, K\right)$ denote the chain complex of $\widehat{C F}\left(S^{3}\right)$, together with this $\mathbb{Z}$-filtration induced from $K$.

Since there are only finitely many generators in $X$, the filtration we have defined has finite support; i.e. for all sufficiently small $m \in \mathbb{Z}, \mathcal{F}(K, m)=0$, and for all sufficiently large $m \in \mathbb{Z}, \mathcal{F}(K, m)=\widehat{C F}\left(S^{3}\right)$, and in particular, if $\iota_{K}^{m}$ denotes the map on homology induced by the inclusion $\mathcal{F}(K, m)$ in $\widehat{C F}\left(S^{3}\right)$, then $\iota_{K}^{m}$ is trivial for all sufficiently small $m$, and an isomorphism for all sufficiently large $m$, and hence the quantity $\tau(K)$ defined in the introduction is a finite integer. Although $\tau(K)$ as defined might appear to depend on a choice of particular Heegaard diagram used to define the knot filtration, it is shown in Theorem 3.1 of [17] that in fact the filtered chain homotopy type of the filtered complex $\widehat{C F K}\left(S^{3}, K\right)$ is a knot invariant, and and hence so is the integer $\tau(K)$. (Actually, Theorem 3.1 of [17] is explicitly stated for the induced filtration of $C F^{\infty}$, which generalizes the filtration of $\widehat{C F}$ we just described, see also the discussion below.)

Recall that $\widehat{H F}(Y)$ is the simplest of the Heegaard Floer homologies associated to three-manifolds in [15]. There are also induced knot filtrations on the chain complexes associated to the other variants of Heegaard Floer homology. Although these filtrations are not used in the definition of $\tau$, they are used in the verification of its four-dimensional properties.

To this end, recall that in [15], there is another invariant for integer homology three-spheres $Y, C F^{\infty}(Y)$, whose generators are pairs $[\mathbf{x}, i] \in\left(\mathbb{T}_{\alpha} \cap \mathbb{T}_{\beta}\right) \times \mathbb{Z}$, endowed with a differential given by the formula

$$
\partial[\mathbf{x}, i]=\sum_{\mathbf{y} \in \mathbb{T}_{\alpha} \cap \mathbb{T}_{\beta}} \sum_{\left\{\phi \in \pi_{2}(\mathbf{x}, \mathbf{y})\right\}} \#\left(\frac{\mathcal{M}(\phi)}{\mathbb{R}}\right)\left[\mathbf{y}, i-n_{w}(\phi)\right],
$$


where here $\pi_{2}(\mathbf{x}, \mathbf{y})$ denotes the space of homotopy classes of Whitney disks in $\operatorname{Sym}^{g}(S)$ connecting $\mathbf{x}$ to $\mathbf{y}$, and $\mathcal{M}(\phi)$ denotes the moduli space of pseudoholomorphic representatives for $\phi$, and in fact, the coefficient of $\left[\mathbf{y}, i-n_{w}(\phi)\right]$ is a suitable signed count of points in the moduli space, after we divide out by the one-dimensional automorphism group of the unit disk in the complex plane, fixing $\sqrt{-1}$ and $-\sqrt{-1}$. This chain complex admits a subcomplex $C F^{-}(Y)$, generated by those pairs $[\mathbf{x}, i]$ with $i<0$, and a quotient complex $C F^{+}(Y)$, which can be thought of as generated by pairs $[\mathbf{x}, i]$ with $i \geq 0$. There is an endomorphism $U$ of the chain complex $C F^{\infty}(Y)$, which respects the subcomplex $C F^{-}(Y)$, defined by $U[\mathbf{x}, i]=[\mathbf{x}, i-1]$.

Indeed, the same definition can be made for rational homology spheres. In this case, there is an identification $\mathfrak{s}_{w}: X \longrightarrow \operatorname{Spin}^{c}(Y)$, and a corresponding splitting of complexes

$$
C F^{\infty}(Y) \cong \bigoplus_{\mathfrak{s} \in \operatorname{Spin}^{c}(Y)} C F^{\infty}(Y, \mathfrak{s}) .
$$

Extra care is to be taken in the case where $b_{1}(Y)>0$, but we do not describe this here, as such three-manifolds do not play a role in the present article. The main result from [15] states that the homology groups $H F^{-}(Y, \mathfrak{s}), H F^{\infty}(Y, \mathfrak{s})$, and $H^{+}(Y, \mathfrak{s})$, thought of as modules over $\mathbb{Z}[U]$, are topological invariants of the three-manifold $Y$ and its Spin $^{c}$ structure $\mathfrak{s}$. In fact, the long exact sequences associated to the two short exact sequences

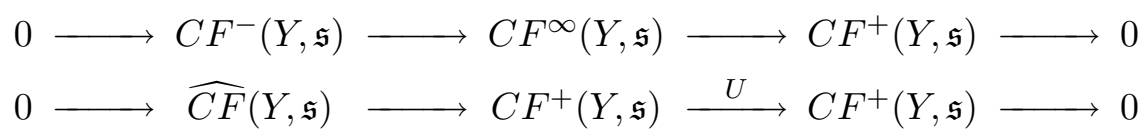

are also topological invariants.

A knot $K \subset S^{3}$ induces a $\mathbb{Z} \oplus \mathbb{Z}$ filtration of $C F^{\infty}\left(S^{3}\right)$ (and also $C F^{-}\left(S^{3}\right)$ and $\left.C F^{+}\left(S^{3}\right)\right)$, as follows. Let $C F K^{\infty}\left(S^{3}, K\right)$ denote the complex generated by $[\mathbf{x}, i, j] \in\left(\mathbb{T}_{\alpha} \cap \mathbb{T}_{\beta}\right) \times \mathbb{Z} \times \mathbb{Z}$ with the property that $\mathcal{F}(\mathbf{x})+(i-j)=0$, and differential

$$
\partial[\mathbf{x}, i, j]=\sum_{\mathbf{y} \in \mathbb{T}_{\alpha} \cap \mathbb{T}_{\beta}} \sum_{\left\{\phi \in \pi_{2}(\mathbf{x}, \mathbf{y})\right\}} \#\left(\frac{\mathcal{M}(\phi)}{\mathbb{R}}\right)\left[\mathbf{y}, i-n_{w}(\phi), j-n_{z}(\phi)\right]
$$

(where, once again, we use some doubly-pointed Heegaard diagram compatible with the knot). The map which which associates to $[\mathbf{x}, i, j]$ the pair $(i, j) \in \mathbb{Z} \oplus \mathbb{Z}$ induces a $\mathbb{Z} \oplus \mathbb{Z}$-filtration on $C F K^{\infty}\left(S^{3}, K\right)$, meaning that if $[\mathbf{y}, k, \ell]$ appears in $\partial[\mathbf{x}, i, j]$ with a non-zero coefficient, then $k \leq i$ and $\ell \leq j$. There is an endomorphism of $C F K^{\infty}\left(S^{3}, K\right)$ defined by $U \cdot[\mathbf{x}, i, j]=[\mathbf{x}, i-1, j-1]$. The forgetful map sending $[\mathbf{x}, i, j]$ to $[\mathbf{x}, i]$ induces an isomorphism of chain complexes $C F K^{\infty}\left(S^{3}, K\right)$ and $C F^{\infty}\left(S^{3}\right)$. Moreover, this map is $\mathbb{Z}[U]$-equivariant. 


\section{$2.2 \quad$ Knot filtrations and surgeries}

Let $K \subset Y$ be a knot with framing $\lambda$ in a three-manifold, and let $X_{\lambda}(K)$ denote the four-manifold obtained by attaching a two-handle to $[0,1] \times Y$ to $\{1\} \times Y$ along $K$ with framing $\lambda$. This can be thought of as a cobordism from $Y$ to the three-manifold $Y_{\lambda}(K)$ obtained by performing $\lambda$-framed surgery on $Y$ along $K$. Given an $\operatorname{Spin}^{c}$ structure $\mathfrak{s}$ on $X_{\lambda}(K)$, there is an induced map

$$
\widehat{F}_{W, \mathfrak{s}}: \widehat{H F}\left(Y,\left.\mathfrak{s}\right|_{Y}\right) \longrightarrow \widehat{H F}\left(Y_{\lambda}(K),\left.\mathfrak{s}\right|_{Y_{\lambda}(K)}\right),
$$

(and also maps for the other versions of Floer homology). This map is induced from a corresponding chain map, gotten by counting pseudo-holomorphic triangles in $\operatorname{Sym}^{g}(\Sigma)$, as explained in Section 9 of [14]. Further invariance properties of these maps, and a generalization to other cobordisms, are established in [11].

In Section 4 of [17], we described the relationship between this knot filtration and the Heegaard Floer homologies of three-manifolds obtained by performing "sufficiently large" integral surgeries on $S^{3}$ along $K$. We sketch the results here, and refer the reader to [17] for a more thorough treatment. Moreover, this relationship gives an interpretation of the some of maps induced by cobordisms in terms of the knot filtration.

Let $K \subset S^{3}$ be a knot. We write simply $C$ for the $\mathbb{Z} \oplus \mathbb{Z}$ filtered complex $C F K^{\infty}\left(S^{3}, K\right)$, suppressing the knot from the notation. The subcomplex $C F^{-}\left(S^{3}\right)$ is represented by the complex $C\{i<0\}$. (This notation means that the complex in question is the subcomplex of $C$ generated by those homogeneous elements whose $\mathbb{Z} \oplus \mathbb{Z}$ filtration level $(i, j)$ satisfies the stated constraint). Its quotient $C F^{+}\left(S^{3}\right)$ is represented by $C\{i \geq 0\}$. The subcomplex $\widehat{C F}\left(S^{3}\right) \subset C F^{+}\left(S^{3}\right)$ is represented by $C\{i=0\}$, and the various filtration levels $\mathcal{F}(K, m)$ correspond to the subcomplexes $C\{i=0, j \leq m\}$ of $C\{i=0\}$ (i.e. the subcomplex generated $[\mathbf{x}, i, j]$ where with $i=0$ and $j \leq m$ ).

Framings on knots in $S^{3}$ are canonically identified with the integers. For given $n \in \mathbb{Z}$, on the three-manifold $S_{-n}^{3}(K)$ obtained by $-n$-framed surgery on $S^{3}$ along $K$, there is a natural affine identification $\operatorname{Spin}^{c}\left(S_{-n}^{3}(K)\right) \cong \mathbb{Z} / n \mathbb{Z}$, specified by an orientation for the knot $K$. More precisely, an orientation of $K$ induces an orientation on its Seifert surface $\Sigma$. The oriented Seifert surface $\Sigma$ can be capped off inside the two-handle to obtain a closed surface $\widehat{\Sigma}$ inside the cobordism $X_{-n}(K)$ from $S^{3}$ to $S_{-n}^{3}(K)$ obtained by the two-handle addition. Now, for fixed $m \in \mathbb{Z}$, consider the $\operatorname{Spin}^{c}$ structure $\mathfrak{s}_{m} \in \operatorname{Spin}^{c}\left(X_{-n}(K)\right)$ with the property that

$$
\left\langle c_{1}\left(\mathfrak{s}_{m}\right),[\widehat{\Sigma}]\right\rangle-n=2 m .
$$


Restricting to $S_{-n}^{3}(K) \subset \partial X_{-n}(K)$, we get the induced identification

$$
\operatorname{Spin}^{c}\left(S_{-n}^{3}(K)\right) \cong \mathbb{Z} / n \mathbb{Z} \text {. }
$$

For $[m] \in \mathbb{Z} / n \mathbb{Z}$, we let $\widehat{H F}\left(S_{-n}^{3}(K),[m]\right)$ denote the summand of the Floer homology corresponding to the Spin ${ }^{c}$ structure corresponding to $[m]$. (Although the orientation on $K$ might appear important here, the other choice of orientation induces a conjugate identification of $\operatorname{Spin}^{c}$ structures, which gives an isomorphic theory, cf. Proposition 3.9 of [17].)

Theorem 4.1 of [17] states that for each integer $m \in \mathbb{Z}$, there is an integer $N$ so that for all $n \geq N$, the short exact sequence of complexes

$$
0 \rightarrow C F^{-}\left(S_{-n}^{3}(K),[m]\right) \longrightarrow C F^{\infty}\left(S_{-n}^{3}(K),[m]\right) \longrightarrow C F^{+}\left(S_{-n}^{3}(K),[m]\right) \rightarrow 0
$$

is identified with the natural short exact sequence

$$
0 \longrightarrow C\{\min (i, j-m)<0\} \longrightarrow C \longrightarrow C\{\min (i, j-m) \geq 0\} \longrightarrow 0 .
$$

Similarly, the inclusion

$$
0 \longrightarrow \widehat{C F}\left(S_{-n}^{3}(K),[m]\right) \longrightarrow C F^{+}\left(S_{-n}^{3}(K),[m]\right)
$$

is identified with the inclusion

$$
0 \longrightarrow C\{\min (i, j-m)=0\} \longrightarrow C\{\min (i, j-m) \geq 0\} .
$$

(In the interest of simplicity, we will remain in the context of $\widehat{H F}$ as much as possible, and in fact will not to use Theorem 4.1 for the theories $H F^{-}, H F^{\infty}$, and $H F^{+}$.)

There is a natural chain map $C\{i=0\} \longrightarrow C\{\min (i, j-m)=0\}$, which is defined to vanish in the subcomplex of $C\{i=0, j<m\}$. In fact, the proof of Theorem 4.1 of [17] shows that this chain map induces the map from $\widehat{H F}\left(S^{3}\right)$ to $\widehat{H F}\left(S_{-n}^{3}(K),[m]\right)$ given by the two-handle addition, endowed with the $\operatorname{Spin}^{c}$ structure for which

$$
\left\langle c_{1}(\mathfrak{s}),[\widehat{\Sigma}]\right\rangle-n=2 m
$$

(again, provided that $n$ is sufficiently large compared to $m$ and the genus of the knot).

Note that a similar picture holds for positive integral surgeries (cf. Theorem 4.1), but we do not need that statement here. 


\section{Proof of Theorem 1.1}

Our aim here is to prove Theorem 1.1. As a preliminary step, we establish a four-dimensional interpretation of $\tau$ which will be useful later. Then, we establish some a priori properties of $\tau$ : additivity under connected sums, and its behavior under reflection. As a third step, we describe some properties of the maps induced on $\widehat{H F}$ by cobordisms (analogous to the usual adjunction inequalities of four-manifold invariants). After establishing these preliminaries, we prove Theorem 1.1. Proofs of its corollaries are given in the end of the section.

\subsection{A four-dimensional interpretation of $\tau$}

The following interpretation of $\tau$ will be useful to us.

But first, we set up notation. For $m \in \mathbb{Z}$, we have a short exact sequence

$$
0 \longrightarrow \mathcal{F}(K, m) \stackrel{I_{K}^{m}}{\longrightarrow} \widehat{C F}\left(S^{3}\right) \stackrel{P_{K}^{m}}{\longrightarrow} Q(K, m) \longrightarrow 0,
$$

where $I_{K}^{m}$ is the natural inclusion, and $P_{K}^{m}$ and $Q(K, m)$ are are defined to make the sequence exact. Let $\iota_{K}^{m}$ and $p_{K}^{m}$ denote the maps induced by $I_{K}^{m}$ and $P_{K}^{m}$ on homology. Of course, $I_{K}^{m}$ is represented by the inclusion of $C\{i=0, j \leq m\}$ inside $C\{i=0\}$.

Fix a knot $K \subset S^{3}$, and an integer $n$. Let

$$
\widehat{F}_{n, m}: \widehat{H F}\left(S^{3}\right) \longrightarrow \widehat{H F}\left(S_{-n}^{3}(K),[m]\right)
$$

denote the map associated to the two-handle addition, endowed with the $\operatorname{Spin}^{c}$ structure $\mathfrak{s}_{m}$ characterized by

$$
\left\langle c_{1}\left(\mathfrak{s}_{m}\right),[\widehat{\Sigma}]\right\rangle-n=2 m .
$$

Proposition 3.1 If $m<\tau(K)$, then $\widehat{F}_{n, m}$ is non-trivial for all sufficiently large $n$. Also, if $m>\tau(K)$, then $\widehat{F}_{n, m}$ is trivial for all sufficiently large $n$.

Proof Consider the diagram

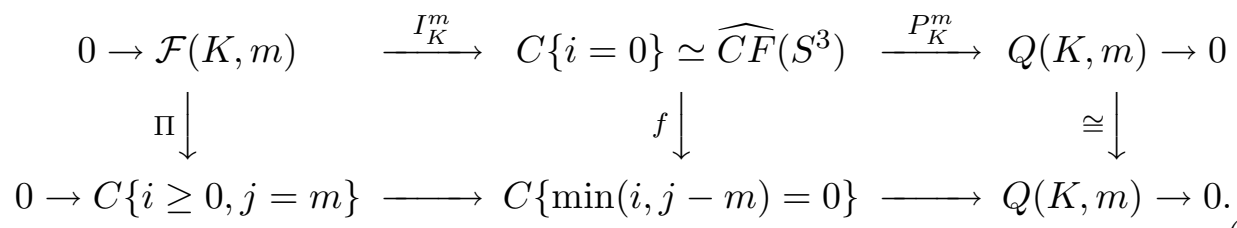


According to Theorem 4.1 of [17] for $n$ sufficiently large, we have an identification

$$
C\{\min (i, j-m)=0\} \simeq \widehat{C F}\left(S_{-n}^{3}(K),[m]\right)
$$

under which the map $f$ represents the chain map $\widehat{F}_{n, m}$ above. Now, if $m<$ $\tau(K)$, the induced map on homology map $p_{K}^{m}$ induces an injection in homology, and hence $\widehat{F}_{m, n}$ is non-trivial. Moreover, since the projection $f$ is trivial on

$$
C\{i=0, j \leq m-1\}=\mathcal{F}(K, m-1),
$$

$\widehat{F}_{n, m}$ factors through the map $P_{K}^{m-1}$. If $m>\tau(K)$, the induced map $p_{K}^{m-1}$ on homology is trivial, and hence so is $\widehat{F}_{n, m}$.

\subsection{Additivity of $\tau$}

The additivity of $\tau$ under connected sums follows readily from the Künneth principle for the knot filtration, Theorem 7.1 of [17].

The maps $I_{K}^{m}$ induces a filtration of $\widehat{C F}\left(S^{3}\right) \otimes_{\mathbb{Z}} \widehat{C F}\left(S^{3}\right)$ as the image of

$$
\sum_{m_{1}+m_{2}=m} I_{K_{1}}^{m_{1}} \otimes I_{K_{2}}^{m_{2}}: \bigoplus_{m_{1}+m_{2}=m} \mathcal{F}\left(K_{1}, m_{1}\right) \otimes_{\mathbb{Z}} \mathcal{F}\left(K_{2}, m_{2}\right) \longrightarrow \widehat{C F}\left(S^{3}\right) \otimes_{\mathbb{Z}} \widehat{C F}\left(S^{3}\right) .
$$

According to Theorem 7.1 of [17], under a homotopy equivalence $\widehat{C F}\left(S^{3}\right) \otimes_{\mathbb{Z}}$ $\widehat{C F}\left(S^{3}\right) \simeq \widehat{C F}\left(S^{3}\right)$, the above filtration is identified with the filtration of $\widehat{C F}\left(S^{3}\right)$ induced by the connected sum $K_{1} \# K_{2}$.

Indeed, for simplicity of exposition, we switch from base ring $\mathbb{Z}$ to $\mathbb{Q}$, a change which we also suppress from the notation. In this case, then, we can think of $\tau(K)$ as the minimum integer for which

$$
\iota_{K}^{m}: H_{*}(\mathcal{F}(K, m)) \longrightarrow \widehat{H F}\left(S^{3}\right) \cong \mathbb{Q}
$$

is surjective.

Proposition 3.2 Let $K_{1}$ and $K_{2}$ be a pair of knots in $S^{3}$, and let $K_{1} \# K_{2}$ denote their connected sum. Then,

$$
\tau\left(K_{1} \# K_{2}\right)=\tau\left(K_{1}\right)+\tau\left(K_{2}\right) .
$$

Proof According to the theorem quoted above, $I_{K_{1} \# K_{2}}^{m}$ is surjective on homology if and only if there is a decomposition $m=m_{1}+m_{2}$ with the property that

$$
f=\left(I_{K_{1}}^{m_{1}} \otimes_{\mathbb{Q}} I_{K_{2}}^{m_{2}}\right)_{*}: H_{*}\left(\mathcal{F}\left(K_{1}, m_{1}\right) \otimes_{\mathbb{Q}} \mathcal{F}\left(K_{2}, m_{2}\right)\right) \longrightarrow \widehat{H F}\left(S^{3}\right) \cong \mathbb{Q}
$$


is surjective. Now, by the Künneth formula, we have an identification

$$
H_{*}\left(\mathcal{F}\left(K_{1}, m_{1}\right)\right) \otimes_{\mathbb{Q}} H_{*}\left(\mathcal{F}\left(K_{2}, m_{2}\right)\right) \cong H_{*}\left(\mathcal{F}\left(K_{1}, m_{1}\right) \otimes_{\mathbb{Q}} \mathcal{F}\left(K_{2}, m_{2}\right)\right),
$$

and hence the map $f$ is surjective if and only if

$$
\begin{aligned}
& \iota_{K_{1}}^{m_{1}} \otimes_{\mathbb{Q}} \iota_{K_{2}}^{m_{2}}: H_{*}\left(\mathcal{F}\left(K_{1}, m_{1}\right)\right) \otimes_{\mathbb{Q}} H_{*}\left(\mathcal{F}\left(K_{2}, m_{2}\right)\right) \longrightarrow \widehat{H F}\left(S^{3}\right) \otimes_{\mathbb{Q}} \widehat{H F}\left(S^{3}\right) \\
& \cong \widehat{H F}\left(S^{3}\right) \cong \mathbb{Q}
\end{aligned}
$$

is. This in turn is easily seen to be surjective if and only if both $\iota_{K_{1}}^{m_{1}}$ and $\iota_{K_{2}}^{m_{2}}$ are surjective. This shows that $\tau\left(K_{1} \# K_{2}\right)=\tau\left(K_{1}\right)+\tau\left(K_{2}\right)$.

It is worth pointing out that, strictly speaking, the knot filtration depends on an oriented knot. However, conjugation invariance of the knot filtration shows that $\tau(K)$ is independent of this additional choice (cf. Proposition 3.8 of [17]).

We have also the following result:

Lemma 3.3 Let $K$ be a knot, and let $-K$ denote its reflection. Then,

$$
\tau(-K)=-\tau(K) .
$$

Proof Let $I_{K}^{m}$ be as before, and indeed we have a short exact sequence

$$
0 \longrightarrow \mathcal{F}(K, m) \stackrel{I_{K}^{m}}{\longrightarrow} \widehat{C F}\left(S^{3}\right) \stackrel{P_{K}^{m}}{\longrightarrow} Q(K, m) \longrightarrow 0 .
$$

According to [14], there is a duality map

$$
\mathcal{D}: \widehat{H F}_{*}\left(S^{3}\right) \longrightarrow \widehat{H F}^{*}\left(-S^{3}\right),
$$

induced by a map of chain complexes, which we also denote by

$$
\mathcal{D}: \widehat{C F}_{*}\left(S^{3}\right) \longrightarrow \widehat{C F}^{*}\left(-S^{3}\right) \text {. }
$$

It is easy to see that under this map, if $K \subset S^{3}$ is a knot, then we have the commutative diagram

$$
\begin{array}{ccc}
\mathcal{F}(K, m) & \stackrel{I_{K}^{m}}{\longrightarrow} \widehat{C F}_{*}\left(S^{3}\right) \\
\mathcal{D} \downarrow \cong & \cong \downarrow \mathcal{D} \\
Q^{*}(-K,-m) & \stackrel{P_{-m}^{-K}}{\longrightarrow} \widehat{C F}^{*}\left(S^{3}\right),
\end{array}
$$

where $P_{-K}^{-m}$ is the map which is dual to $P_{-K}^{-m}$. The induced map on cohomology $p_{-K}^{-m}$ is trivial if and only if othe map $\iota_{-K}^{-m}$ is non-trivial. The lemma now follows. 


\subsection{Maps on $\widehat{H F}$}

We turn now to some more lemmas which will be used in the proof of Theorem 1.1. But first, we must set up some more notation.

Let $W$ be a four-manifold with $b_{2}^{+}(W)=0=b_{1}(W)$ and $\partial W=S^{3}$, and let $K \subset S^{3}$ be a knot. Let $W_{-n}(K)$ denote the four-manifold obtained by attaching a two-handle to $W$ along $K$, with framing $-n$. As in the statement of Theorem 1.1, we fix a surface $\Sigma$ whose boundary lies in $\partial W$, where it agrees with the knot $K$. This surface-with-boundary can be closed off to obtain a smoothly embedded surface $\widehat{\Sigma}$ inside $W_{-n}(K)$.

Then, we can view $W-B^{4}$ as a cobordism from $S^{3}$ to $S^{3}$. According to Donaldson's theorem, $W$ has diagonalizable intersection form. Thus, if we let $b=b_{2}(W)$, there are are $2^{b}$ characteristic vectors $K$ for the intersection form (on $H^{2}(W ; \mathbb{Z}) /$ Tors) with $K \cdot K=-b$. Note that if $[\Sigma] \in H_{2}(W ; \mathbb{Z})$, then

$$
|[\Sigma]|=\max _{\{K \in \operatorname{Char}(W) \mid K \cdot K=-b\}}\langle K,[\Sigma]\rangle,
$$

where $\operatorname{Char}(W) \subset H^{2}(W ; \mathbb{Z}) /$ Tors denotes the set of characteristic vectors for the intersection form.

Note that in $W_{-n}(K)$,

$$
[\widehat{\Sigma}] \cdot[\widehat{\Sigma}]=[\Sigma] \cdot[\Sigma]-n
$$

Lemma 3.4 Let $\mathfrak{s}$ be a $\operatorname{Spin}^{c}$ structure over a four-manifold $W$ with $\partial W=$ $S^{3}, b_{2}^{+}(W)=0$, and $b_{2}(W)=b$. Then its first Chern class satisfies

$$
c_{1}(\mathfrak{s}) \cdot c_{1}(\mathfrak{s})=-b
$$

if and only if the induced map

$$
\widehat{F}_{W-B, \mathfrak{s}}: \widehat{H F}\left(S^{3}\right) \longrightarrow \widehat{H F}\left(S^{3}\right)
$$

is non-trivial (in which case it is an isomorphism).

Proof In [18] (see especially the proof of Theorem 9.6 of [18]), it is shown that the map induced by $W-B^{4}$ on $H F^{\infty}$ is an isomorphism, and its shift in degree is given by

$$
\left(c_{1}(\mathfrak{s})^{2}+b\right) / 4 \text {. }
$$

Thus, the map on $\mathrm{HF}^{+}$induced by a $\operatorname{Spin}^{c}$ structure $\mathfrak{s}$ is an isomorphism if and only if Equation (5) holds. The lemma now follows readily from the long exact sequence relating $\widehat{H F}$ and $H F^{+}$(cf. Equation (1)), and its functoriality under the maps induced by cobordisms (cf. [11]). 
Lemma 3.5 Let $N$ be the total space of circle bundle with Euler number $-n<0$ over an oriented two-manifold $\Sigma$ of genus $g>0$. The map

$$
\widehat{F}_{N-B, \mathfrak{s}}: \widehat{H F}\left(S^{3}\right) \longrightarrow \widehat{H F}(\partial N)
$$

is trivial whenever

$$
\left\langle c_{1}(\mathfrak{s}),[\Sigma]\right\rangle+[\Sigma] \cdot[\Sigma]>2 g(\Sigma)-2 .
$$

Proof We argue as in [16] and [13], making use of the absolute grading on Floer homology. Specifically, according to Theorem 7.1 of [11], there is an absolute $\mathbb{Q}$-grading on the Floer homology $\widehat{H F}$ of any three-manifold equipped with a torsion $\operatorname{Spin}^{c}$ structure, which is uniquely characterized by the following two properties: $\widehat{H F}\left(S^{3}\right)$ is supported in dimension zero, and if $W$ is a cobordism from $Y_{1}$ to $Y_{2}$, which is given a $\operatorname{Spin}^{c}$ structure $\mathfrak{s}$ whose restrictions to $Y_{1}$ and $Y_{2}$ have torsion first Chern class, then the induced map

$$
\widehat{F}_{W, \mathfrak{s}}: \widehat{H F}\left(Y_{1},\left.\mathfrak{s}\right|_{Y_{1}}\right) \longrightarrow \widehat{H F}\left(Y_{2},\left.\mathfrak{s}\right|_{Y_{2}}\right)
$$

shifts degree by

$$
\frac{c_{1}(\mathfrak{s})^{2}-2 \chi(W)-3 \sigma(W)}{4} .
$$

With respect to this absolute grading, the rank of $\widehat{H F}_{i}\left(\#^{2 g}\left(S^{2} \times S^{1}\right)\right)$ is zero if $|i|>g$. The Heegaard Floer homology of $\#^{2 g}\left(S^{2} \times S^{1}\right)$ can be calculated directly from its Heegaard diagram, cf. Subsection 9.1 of [15]. Indeed there is a constant $c$ with the property that $\widehat{H F}_{i+c}\left(\#^{2 g}\left(S^{2} \times S^{1}\right)\right)$ is a free module whose rank is given by the binomial coefficient $\left(\begin{array}{c}2 g \\ i\end{array}\right)$. (We suppress $\operatorname{Spin}^{c}$ structures from the notation for $\widehat{H F}$ of $\#^{2 g}\left(S^{2} \times S^{1}\right)$, since that is non-trivial only in the $\operatorname{Spin}^{c}$ structure with trivial first Chern class; the subscript $i$ here denotes the absolute $\mathbb{Q}$-degree). The stated vanishing follows from the fact that $c=g$. To see this, note that a direct inspection of the Heegaard diagrams shows that the non-zero elements in $\widehat{H F}\left(\#^{2 g}\left(S^{2} \times S^{1}\right)\right)$ with lowest degree are in the image of the map on $\widehat{H F}$ induced by the cobordism $S^{3}$ to $\#^{2 g}\left(S^{2} \times S^{1}\right)$ obtained by attaching $2 g$ two-handles. In turn this cobordism shifts degrees down by $g$, according to Equation (6).

Now, by blowing up $N$ sufficiently many times (and using the "blow-up formula" of [11]), we reduce to the case where $N$ is the total space of a circle bundle with $-n<-2 g+1<0$. In this case,

$$
\widehat{H F}(\partial N, \mathfrak{s} \mid \partial N) \cong \widehat{H F}\left(\#^{2 g}\left(S^{2} \times S^{1}\right)\right) \text {, }
$$


as relatively $\mathbb{Z}$-graded Abelian groups, as can be seen by appealing to the long exact sequence for integral surgeries. In fact, letting $\mathfrak{s}_{0}$ denote the Spin ${ }^{c}$ structure which minimizes $c_{1}\left(\mathfrak{s}_{0}\right)^{2}$ among all $\operatorname{Spin}^{c}$ structures $\mathfrak{s}^{\prime}$ over $N$ which have $\left.\mathfrak{s}^{\prime}\right|_{\partial N}=\left.\mathfrak{s}\right|_{\partial N}$, it is the map induced by $\mathfrak{s}_{0}$, which shifts absolute degree by $\frac{c_{1}\left(\mathfrak{s}_{0}\right)^{2}+1}{4}$, which induces the isomorphism of Equation (7):

$$
\widehat{F}_{W, \mathfrak{s}_{0} \mid W}: \widehat{H F}\left(\#^{2 g}\left(S^{2} \times S^{1}\right)\right) \stackrel{\cong}{\longrightarrow} \widehat{H F}(\partial N, \mathfrak{s} \mid \partial N) .
$$

Here we have broken $N$ into one zero handle, $2 g$ one-handles, and one twohandle; it is the latter which specifies the cobordism $W$ from $\#^{2 g}\left(S^{2} \times S^{1}\right)$ to $\partial N$. (Essentially this calculation, with more details, can be found in Lemma 9.17 of [18], only there we consider the case of $H F^{+}$, rather than $\widehat{H F}$.)

The stated hypothesis on $\mathfrak{s}$, together with the fact that $\widehat{H F}\left(\#^{2 g}\left(S^{2} \times S^{1}\right)\right)$ is supported in degrees $[-g, g]$ shows now that the map induced by $\mathfrak{s}$ is trivial.

\subsection{Proof of Theorem 1.1}

Let $W$ and $\Sigma$ be as in the statement of the theorem. We subdivide the proof into two cases: $g(\Sigma)=g>0$ and $g=0$.

Proof of Theorem 1.1 when $g>0$ For an integer $n$ (which we will fix later), we let $W^{\prime}$ denote the four-manifold obtained by deleting an open fourball from the interior of four-manifold $W_{-n}(K)$ (which is disjoint from $\Sigma$ ). This four-manifold decomposes as

$$
W^{\prime} \cong W_{1} \cup_{S^{3}} W_{2},
$$

where $W_{1}$ is obtained by deleting a small four-ball from $W$ (and hence it is independent of $n$ ), while $W_{2}=X_{-n}(K)$ is the cobordism from $S^{3}$ to $S_{-n}^{3}(K)$ specified by the two-handle addition. We close off $\Sigma$ inside the two-handle to obtain an surface $\widehat{\Sigma}$ with $g(\widehat{\Sigma})=g(\Sigma)$. Moreover, we can split the homology class $[\widehat{\Sigma}]=\left[\Sigma_{1}\right] \oplus\left[\Sigma_{2}\right]$, where $\left[\Sigma_{i}\right] \in H_{2}\left(W_{i} ; \mathbb{Z}\right)$. Note that

$$
\left|\left[\Sigma_{1}\right]\right|=|[\Sigma]| \text { and }[\Sigma] \cdot[\Sigma]=\left[\Sigma_{1}\right] \cdot\left[\Sigma_{1}\right] \text {. }
$$

Fix a $\operatorname{Spin}^{c}$ structure $\mathfrak{s}_{1} \in \operatorname{Spin}^{c}\left(W_{1}\right)$ so that

$$
c_{1}\left(\mathfrak{s}_{1}\right)^{2}+b=0 \quad \text { and } \quad\left|\left[\Sigma_{1}\right]\right|=\left\langle c_{1}\left(\mathfrak{s}_{1}\right),\left[\Sigma_{1}\right]\right\rangle .
$$

For any integer $m<\tau(K)$, we choose $n$ large enough that Proposition 3.1 holds, and fix a Spin ${ }^{c}$ structure $\mathfrak{s}_{2}$ over $W_{2}$ so that

$$
\left\langle c_{1}\left(\mathfrak{s}_{2}\right),\left[\Sigma_{2}\right]\right\rangle-n=2 m<2 \tau(K) .
$$


According to Lemma 3.4 and Proposition 3.1 respectively, the maps $\widehat{F}_{W_{1}, \mathfrak{s}_{1}}$ and $\widehat{F}_{W_{2}, \mathfrak{s}_{2}}$ induce non-trivial maps on $\widehat{H F}$. Thus, by naturality of the maps induced by cobordisms (cf. [15]), if we let $\mathfrak{s}$ be a $\operatorname{Spin}^{c}$ structure with $\mathfrak{s} \mid W_{i}=\mathfrak{s}_{i}$, the map $\widehat{F}_{W^{\prime}, \mathfrak{s}}$ induces a non-trivial map on $\widehat{H F}$.

Note that $\widehat{\Sigma}$ is represented by a closed, embedded surface of genus $g$, so we can split the cobordism $W^{\prime}$ alternately as $W_{1}^{\prime} \cup W_{2}^{\prime}$, where $W_{1}^{\prime}$ is the tubular neighborhood of $\widehat{\Sigma}$ minus a four-ball (thought of as a subset of $W^{\prime}$, containing one of its boundary components), and $W_{2}^{\prime}$ is the remaining part of $W^{\prime}$.

It now follows from Lemma 3.5 that

$$
\left\langle c_{1}(\mathfrak{s}),[\widehat{\Sigma}]\right\rangle+[\widehat{\Sigma}] \cdot[\widehat{\Sigma}] \leq 2 g-2 .
$$

On the other hand, it is easy to see that the left-hand-side is

$$
\left|\left[\Sigma_{1}\right]\right|+\left[\Sigma_{1}\right] \cdot\left[\Sigma_{1}\right]+\left\langle c_{1}(\mathfrak{s}),\left[\Sigma_{2}\right]\right\rangle+\left[\Sigma_{2}\right] \cdot\left[\Sigma_{2}\right]=\left|\left[\Sigma_{1}\right]\right|+\left[\Sigma_{1}\right] \cdot\left[\Sigma_{1}\right]+2 m .
$$

It follows at once that if $g>0$, then

$$
2 \tau(K)+|[\Sigma]|+[\Sigma] \cdot[\Sigma] \leq g(\Sigma) .
$$

Proof of Theorem 1.1 when $g=0$ Fixing $W, K$, and $\Sigma$ as before, except now we assume that $\Sigma$ is a disk. We can form a new four-manifold $W \#_{b} W$ by boundary connected sum, which contains $K \# K$ on its boundary, a knot which bounds the smoothly-embedded disk $\Sigma^{\prime}=\Sigma \#_{b} \Sigma$. Adding a trivial handle to $\Sigma^{\prime}$, and using the previous case of the theorem, we see that

$$
2 \tau(K \# K)+\left|\left[\Sigma^{\prime}\right]\right|+\left[\Sigma^{\prime}\right] \cdot\left[\Sigma^{\prime}\right] \leq 2 .
$$

According to Proposition 3.2, $\tau(K \# K)=2 \tau(K)$, and it is also easy to see that

$$
\left|\left[\Sigma^{\prime}\right]\right|=2|[\Sigma]| \text { and }\left[\Sigma^{\prime}\right] \cdot\left[\Sigma^{\prime}\right]=2[\Sigma] \cdot[\Sigma] .
$$

Thus, we see that

$$
2 \tau(K)+|[\Sigma]|+[\Sigma] \cdot[\Sigma] \leq 1 .
$$

But the left-hand-side is easily seen to be an even integer, so the stated inequality immediately follows. 


\subsection{Corollaries.}

We now turn to some consequences of Theorem 1.1.

Proof of Corollary 1.3 Apply Theorem 1.1 with $W=B^{4}$, so that $[\Sigma]=0$, to see that $\tau(K) \leq g^{*}(K)$. Reflecting the knot $K$ and applying Lemma 3.3, we get that $-\tau(K) \leq g^{*}(K)$, as well.

Proof of Theorem 1.2 If $K$ is a slice knot (i.e. it bounds a smoothly embedded disk in the four-ball), then $\tau(K)=0$, according to Corollary 1.3. The theorem follows from this fact, together with Proposition 3.2.

Proof of Corollary 1.5 The knot $K=K_{+} \#\left(-K_{-}\right)$clearly bounds an immersed disk in $B^{4}$ with a single double-point. By resolving this double-point, we obtain a smoothly embedded surface with genus 1 in $W=B^{4}$ which bounds $K_{+} \#\left(-K_{-}\right)$, and hence, applying Theorems 1.2 and 1.1,

$$
\tau\left(K_{+}\right)-\tau\left(K_{-}\right) \leq 1 .
$$

This proves one of the two inequalities. For the other inequality, we consider $K_{-} \#\left(-K_{+}\right)$, and observe that if we blow up the self-intersection, we obtain an embedded disk in $W=B^{4} \# \overline{\mathbb{C P}}^{2}$ which represents the trivial homology class. Thus, according to Theorems 1.2 and 1.1, $\tau\left(K_{-}\right)-\tau\left(K_{+}\right) \leq 0$.

\section{Some small examples}

In this section, we calculate $\tau$ for several ten-crossing knots, the knots $10_{139}$, $10_{152}$, and $10_{161}$ from Rolfsen's list [23]. For these knots, the invariant $\tau$ gives sharp lower bounds on the unknotting number and hence the four-ball genus. The four-ball genera of $10_{139}$ and $10_{152}$ were first calculated in [7] and the fourball genus of $10_{161}$ was first calculated by [25]; both results use gauge theory techniques, cf. [8] and [24].

The present calculations rest on the techniques from [19] and their refinements from [20]. These results interpret the generators for $\widehat{C F K}$ in terms of "essential Kauffmann states" for a knot projection. We recall the definitions presently.

Let $K$ be an oriented knot, and let $G$ denote a generic projection for $K$, with distinguished edge $\epsilon_{0}$. This choice of data is called a decorated knot projection G. A Kauffman state (cf. [6]) is an assignment which maps each crossing for the 
knot projection $G$ one of its four adjoining quadrants, so that no two crossings are assigned quadrants from the same region in $S^{2}-G$, and no crossing point is associated to one of the two distinguished regions containing $\epsilon_{0}$.

The chain complex $\widehat{C F K}$ is generated by all Kauffman states for the knot projection. Indeed, there is a smaller complex which can be used when $\epsilon_{0}$ is chosen carefully.

To describe this, we use the notion of an essential interval, cf. [20]. Indeed, it suffices here to consider a slightly weaker notion, which we call a weakly essential interval. A weakly essential interval is a sequence of consecutive edges $E=\bigcup_{i=-\ell}^{m} \epsilon_{i}$ with $\ell, m \geq 0$, so that the following three properties hold:

- $E_{-} \cup \epsilon_{0} \cup E_{+}$is an embedded arc,

- as we traverse $E_{+}$, all the crossings encountered have the same type (i.e. they are over- or under-passes),

- and similarly as we traverse $E_{-}$, all the crossings encountered have the same type (which might be different from the type encountered along $\left.E_{+}\right)$.

If $v$ is a vertex in $E$ (i.e. a crossing for $G$ ), then there are two edges in $E$ which meet $v$ : of these two, one is farther from $\epsilon_{0}$ (in $E$ ). An $E$-essential state is a state which associates to each vertex $v$ in $E$ one of the two regions containing the edge through $v$ which is farther (in $E$ ) from $\epsilon_{0}$.

A Kauffman state $x$ is assigned a filtration level and an absolute grading, according to the rules specified in Figures 1 and 2 respectively.

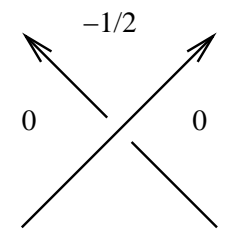

$1 / 2$

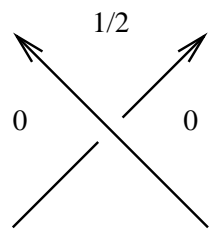

$-1 / 2$

Figure 1: Local filtration level contributions We have illustrated the local contributions for the filtration level of a state for both kinds of crossings.

Finally, if we number all the edges of $G$ consecutively $\left\{\epsilon_{i}\right\}_{i=0}^{N-1}$, we can associate to each state $x$ a multi-filtration-level

$$
M_{x} \in \operatorname{Hom}\left(\left\{\epsilon_{i}\right\}_{i=0}^{N-1}, \mathbb{Z} \oplus \mathbb{Z}\right)
$$



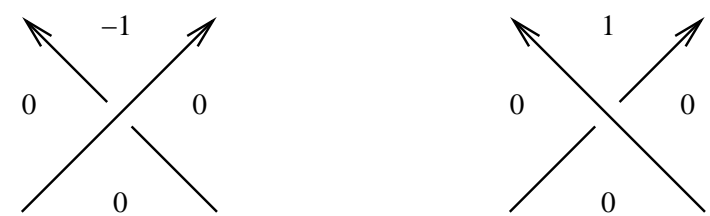

Figure 2: Local grading contributions We have illustrated the local contribution for the absolute grading associated for a state.

by the inductive rules:

$$
M_{x}\left(\epsilon_{i}\right)= \begin{cases}(0,0) & \text { if } i=0 \\ M_{x}\left(\epsilon_{i-1}\right)+(0,1) & \text { if } v_{i} \text { is over and } x\left(v_{i}\right) \text { is to the right } \\ M_{x}\left(\epsilon_{i-1}\right)-(0,1) & \text { if } v_{i} \text { is over and } x\left(v_{i}\right) \text { is to the left } \\ M_{x}\left(\epsilon_{i-1}\right)+(1,0) & \text { if } v_{i} \text { is an under and } x\left(v_{i}\right) \text { is to the left } \\ M_{x}\left(\epsilon_{i-1}\right)-(1,0) & \text { if } v_{i} \text { is an under and } x\left(v_{i}\right) \text { is to the right }\end{cases}
$$

(Here we have abbreviated the conditions. Thus "if $v_{i}$ is over and $x\left(v_{i}\right)$ is to the right" means "if $v_{i}$ is an overcrossing and $x\left(v_{i}\right)$ is to the right of $\epsilon_{i} \cup \epsilon_{i-1}$ " and the other conditions should be expanded similarly.)

The following is a combination of results from [19] and [20]:

Theorem 4.1 Let $G$ be a decorated knot projection for $K$, and a compatible essential interval $E$. Then, there is a chain complex which calculates $\widehat{C F}\left(S^{3}\right)$ whose generators are $E$-essential states, whose filtration levels and absolute gradings are given as above; so, in particular, $\widehat{H F K}\left(S^{3}, i\right)$ is generated by those $E$-essential states with filtration level $i$. Moreover, the differential on $\widehat{C F K}\left(S^{3}\right)$ respects the multi-filtration $M$ defined above, in the sense that if $y$ appears with non-zero multiplicity in $\partial x$, then for each edge $\epsilon$ not in $E$, $M_{x}(\epsilon)-M_{y}(\epsilon)$ is a pair of non-zero integers.

Proof In [19], we describe a Heegaard diagram belonging to the knot projection, for which the generators can be interpreted as Kauffman states, with filtration level and absolute grading calculated above. Indeed, this interpretation is established in Theorem 1.2 of [19], while the restriction to only essential states is described in Proposition 2.6 of [20], with a slight modification of the original Heegaard diagram. Moreover, compatibility with the multi-filtration is established in Proposition 2.8 of [20].

In the decorated knot projections we consider here, there will be a unique maximal essential interval (through $\epsilon_{0}$ ), which we will use as $E$. With this understood, we will drop $E$ from our notation. 


\subsection{The knot $10_{139}$}

Proposition 4.2 For the knot $K=10_{139}$, we have that $g^{*}(K)=u(K)=$ $|\tau(K)|=4$.

Proof In Figure 3, we have given a picture for $K=10_{139}$, circling four crossings. If these four crossings are changed, we obtain a picture of the unknot. Hence, $g^{*}(K) \leq u(K) \leq 4$. Indeed, if we use the indicated decoration for this knot, it is straightforward if tedious to verify that there is a unique essential state in dimension 0 , and it has filtration level 4. (Our calculations were expedited by the use of Mathematica [26].) It follows at once that $\tau(K)=4$. The rest now follows at once from Corollary 1.3.

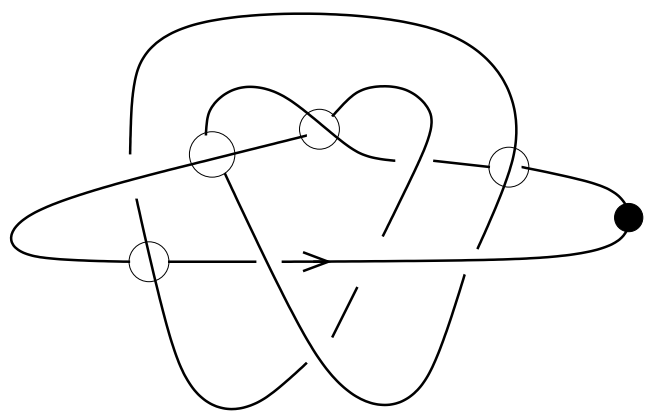

Figure 3: The knot $10_{139}$ We have circled four crossings: when these are switched, the new knot is the unknot. The black dot indicates the distinguished edge $\left(\epsilon_{0}\right)$ used in the decorated knot projection.

\subsection{The knot $10_{152}$}

Proposition 4.3 For the knot $K=10_{152}$, we have that $g^{*}(K)=u(K)=$ $|\tau(K)|=4$.

Proof We proceed exactly as in the proof of Proposition 4.2, only with a different picture. Again, we have indicated the four crossings which are to be changed to obtain the unknot, and we have indicated a distinguished edge $\epsilon_{0}$, with respect to which there is only one essential state in dimension zero, and it has filtration level -4 . Thus $|\tau(K)|=u(K)=g^{*}(K)=4$. 


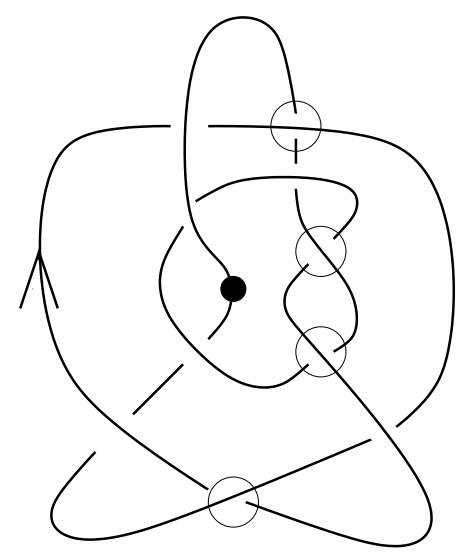

Figure 4: The knot $10_{152}$ We have indicated, once again, the crossings needed to unknot $10_{152}$, and also the distinguished edge $\left(\epsilon_{0}\right.$, with the dark circle) used in the decorated knot projection for the proof of Proposition 4.3.

\subsection{The knot $10_{161}$}

Proposition 4.4 For the knot $K=10_{161}$, we have that $g^{*}(K)=u(K)=$ $|\tau(K)|=3$.

Proof First, observe that the unknotting indicated in Figure 5 shows that $3 \leq u(K) \leq g^{*}(K)$. On the other hand, for the decoration indicated in that figure, there are now exactly two essential states with dimension zero, which we label $a$ and $b$, where $a$ has filtration level -3 and $b$ has filtration level -2 . We claim, however, that there is an essential state $c$ in filtration level -1 with the property that $b$ appears once in the expansion of $\partial c$. The states $c$ and $b$ are illustrated in Figure 6.

To see that $b$ appears once in the expansion of $\partial c$, we consider the decorated knot projection for the trefoil knot illustrated in Figure 7. If we include all states (i.e. we include the inessential ones), then we obtain five states, with the extra two canceling states in filtration level 2 . There remain states $x, y$, and $z$ in filtration levels $-1,0$, and 1 respectively, and absolute degrees 0,1 , and 2 . It follows at once that $\partial z=y$. On the other hand, the support of the domain connecting $z$ to $y$ agrees with the support of the domain connecting $c$ to $b$.

It follows now that either $c$ is null-homologous, or it is homologous to some multiple of $b$. In either case, it follows that $b$ represents a generator of $\widehat{H F}\left(S^{3}\right)$, and hence $\tau(K)=-3$. The result now follows, as usual, from Corollary 1.3. 


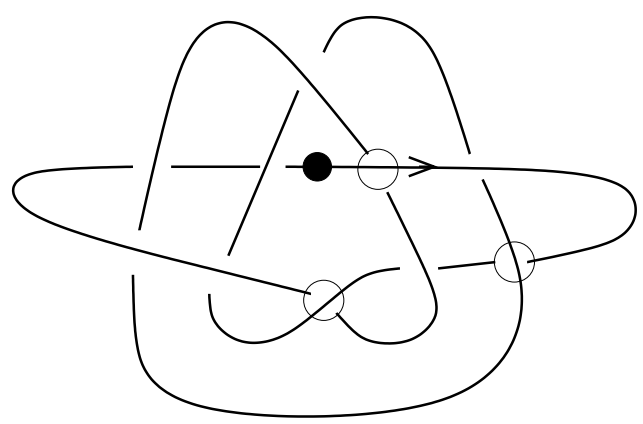

Figure 5: Local filtration level contributions A decorated knot projection for $10_{161}$, showing that it has unknotting number at least 3 .

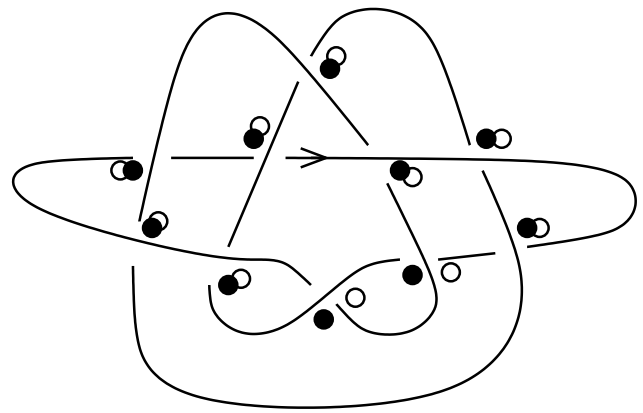

Figure 6: States $b$ and $c$ We have indicated the state $c$ by the dark circles, and $b$ by the hollow ones.

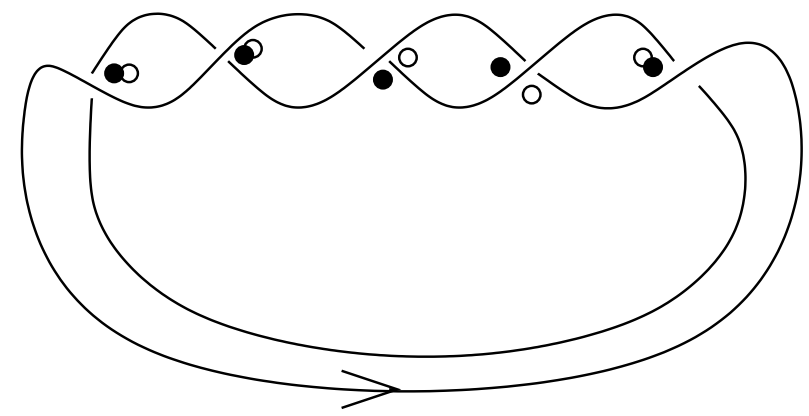

Figure 7: States $y$ and $z$ We have indicated here the two states in absolute degrees 1 and 2. The state $z$ (in dimension two) is indicated by the dark circles, while $y$ is indicated by the hollow ones. It is easy to see that the domain connecting $z$ to $y$ agrees with the domain connecting $c$ to $b$ in the previous picture. 


\section{Generalizations to other three-manifolds}

The constructions of the present paper can, of course, be readily generalized to oriented links in $S^{3}$. Specifically, as in Proposition 2.1 of [17], an oriented, $n$ component link $L$ in $S^{3}$ gives rise to an oriented knot $\kappa(L)$ in $\#^{n-1}\left(S^{2} \times S^{1}\right)$. There is thus an induced filtration

$$
\mathcal{F}(\kappa(L), m) \subset \widehat{C F}\left(\#^{n-1}\left(S^{2} \times S^{1}\right)\right),
$$

and hence maps

$$
\iota_{\kappa(L)}^{m}: H_{*}(\mathcal{F}(\kappa(L), m)) \longrightarrow \widehat{H F}\left(\#^{n-1}\left(S^{2} \times S^{1}\right)\right) \cong \Lambda^{*}\left(\mathbb{Q}^{n-1}\right) .
$$

Again, we define $\tau(L)$ to be the minimum $m$ for which $\iota_{\kappa(L)}^{m}$ is surjective.

In another direction, we can generalize $\tau$ to the case of null-homologous knots in an arbitrary closed, oriented three-manifold $Y$. We focus presently on the case where $Y$ is an integral homology three-sphere. There are natural maps $p: H F^{\infty}(Y) \longrightarrow H F^{+}(Y)$, and $q: \widehat{H F}(Y) \longrightarrow H F^{+}(Y)$. We now let

$$
\tau(Y, K)=\min \left\{m \mid \operatorname{Im}\left(q \circ \iota_{K}^{m}\right) \cap \operatorname{Im}(p) \text { is non-torsion }\right\} .
$$

\section{References}

[1] J O Berge, Some knots with surgeries giving lens spaces, unpublished manuscript

[2] M Boileau, C Weber, Le problème de J. Milnor sur le nombre gordien des noeuds algébriques, Enseign. Math. 30 (1984) 173-222

[3] S K Donaldson, P B Kronheimer, The Geometry of Four-Manifolds, Oxford Mathematical Monographs, Oxford University Press (1990)

[4] C A Giller, A family of links and the Conway calculus, Trans. Amer. Math. Soc. 270 (1982) 75-109

[5] C McA Gordon, R A Litherland, K Murasugi, Signatures of covering links, Canad. J. Math. 33 (1981) 381-394

[6] L H Kauffman, Formal knot theory, Mathematical Notes 30, Princeton University Press (1983)

[7] T Kawamura, The unknotting numbers of $10_{139}$ and $10_{152}$ are 4, Osaka J. Math. 35 (1998) 539-546

[8] P B Kronheimer, TS Mrowka, Gauge theory for embedded surfaces. I, Topology 32 (1993) 773-826

[9] J W Milnor, Singular points of complex hypersurfaces, Annals of Mathematics Studies 61, Princeton University Press (1968) 
[10] B Owens, S Strle, Rational homology spheres and four-ball genus (2003), preprint

[11] P S Ozsváth, Z Szabó, Holomorphic triangles and invariants for smooth fourmanifolds, arXiv:math.SG/0110169

[12] P S Ozsváth, Z Szabó, On knot Floer homology and lens space surgeries, arXiv:math.GT/0303017

[13] P S Ozsváth, Z Szabó, The symplectic Thom conjecture, Ann. of Math. 151 (2000) 93-124

[14] PS Ozsváth, Z Szabó, Holomorphic disks and three-manifold invariants: properties and applications (2001), to appear in Annals of Math. arXiv:math.SG/0105202

[15] P S Ozsváth, Z Szabó, Holomorphic disks and topological invariants for closed three-manifolds (2001), arXiv:math.SG/0101206 To appear in Annals of Math.

[16] P S Ozsváth, Z Szabó, Holomorphic disk invariants for symplectic fourmanifolds (2002), arXiv:math.SG/0201059

[17] P S Ozsváth, Z Szabó, Holomorphic disks and knot invariants (2002), to appear in Adv. in Math.arXiv:math.GT/0209056

[18] P S Ozsváth, Z Szabó, Absolutely graded Floer homologies and intersection forms for four-manifolds with boundary, Adv. Math. 173 (2003) 179-261

[19] P S Ozsváth, Z Szabó, Heegaard Floer homology and alternating knots, Geom. Topol. 7 (2003) 225-254, arXiv:math.GT/0209149

[20] P S Ozsváth, Z Szabó, Knot Floer homology, genus bounds, and mutation (2003), arXiv:math.GT/0303225

[21] J A Rasmussen, Floer homologies of surgeries on two-bridge knots, Algebr. Geom. Topol. 2 (2002) 757-589

[22] J A Rasmussen, Floer homology and knot complements, Ph.D. thesis, Harvard University (2003)

[23] D Rolfsen, Knots and links, Mathematics Lecture Series 7, Publish or Perish, Inc. (1976)

[24] L Rudolph, Qusipositivity as an obstruction to sliceness, Bull. Amer. Math. Soc. (N.S.) 29 (1993) 51-59

[25] T Tanaka, Unknotting numbers of quasipositive knots, Topology Appl. 88 (1998) 239-246

[26] S Wolfram, The Mathematica book. Fourth edition., Wolfram Media, Inc. (1999) 